\title{
A time variational method to couple heterogeneous time integrators
}

\author{
Alain Combescure* — Najib Mahjoubi** — Anthony Gravouil* \\ Nicolas Greffet** \\ * Université de Lyon, INSA de Lyon, LaMCoS UMR CNRS 5259 \\ 18-20 Allée des sciences F-69621 Villeurbanne cedex \\ alain.combescure@insa-lyon.fr \\ ** EDF R\&D (AMA/T62), LaMSID, EDF-CNRS, UMR2832 \\ 1 avenue du Général de Gaulle F-92141 Clamart cedex \\ nicolas.greffet@edf.fr
}

ABSTRACT. This paper is devoted to a brief presentation of recent research results upon structural mechanics code coupling in transient analysis. The domain is supposed to be decomposed into a series of sub domains which are treated independently with their own time integration scheme and or their own code. The paper gives a general method which allows to couple these subdomains. The proposed method is rather general and based upon a weak vision of dynamic equilibrium equation. This new vision allows to design a coupling strategy which ensure by design that no energy is introduced or dissipated in the interfaces between the sub domains. The proposed coupling method hence does not perturb the quality of the time integrators of each sub domain. This also allows to develop a general code coupler for transient dynamics. Two examples are given to illustrate the paper.

RÉSUMÉ. Cet article décrit les derniers résultats de recherche obtenus par l'équipe sur le thème du couplage de codes en dynamique des structures. Le domaine de calcul est découpé en plusieurs sous-domaines qui sont calculés indépendamment chacun avec leur propre algorithme d'intégration temporelle. Ce papier décrit une méthode qui permet de coupler les codes en assurant une nullité stricte de l'énergie d'interface. Le travail repose sur une vision faible de l'intégration temporelle des équations d'équilibre, qui permet de concevoir un algorithme qui vérifie la nullité de l'énergie d'interface. La convergence et la stabilité des algorithmes des sous-domaines sont préservées par le collage proposé.

KEYWORDS: transient dynamics, sub domain coupling, finite elements.

MOTS-CLÉS : dynamique transitoire, couplage de sous-domaines, éléments finis.

DOI:10.3166/EJCM.19.11-24 (C) 2010 Lavoisier, Paris

EJCM - 19/2010. Giens 2009, pages 11 to 24 


\section{Introduction}

The coupling of subdomains is an active research domain these last ten years. After a rather long period of uncertainty on the possibility to develop such a method, the FETI method ((Farhat, 1993),(Farhat, 1994)) showed the importance and interest of dual Schurr methods for sub domain coupling. Alternative methods as the Latin method (Champaney, 1997) (Ladeveze, 2003) use an alternative efficient way to solve transient sub domain couplings using a weak space time vision of the interface gluing : the originality for time variable is the use of a non incremental time integration. The space interfaces are glued using a mixed force and displacement constraint. The Arlequin's method (Dhia, 1998) uses patches which are added onto the coarse mesh to get fine space time solutions within the patch domain. A Gravouil ((Gravouil, 2001), (Combescure, 2002)) extended the FETI concept to show that one could couple sub domains each of them using any Newmark scheme with a perfect energy conservation and he also proved the stability of the coupling algorithm : the key ingredient was the choice to write the coupling equations on the velocity variable. He proved that if the time step is uniform in all the sub domains the interfaces energy is exactly zero which ensures the optimal quality of the coupling. He also showed that when time steps are different in the different sub domains the proposed coupling scheme remains stable but dissipates some energy at the interfaces. The practical examples nevertheless show that this dissipated energy remain small (less than a few \%). This interface dissipation deteriorates the convergence rate of the time integrators in each sub domain. For instance if each subdomain has a second order time integration scheme the coupled problem has only a first order convergence rate. Prakash (Prakash, 2004) recently proposed an improvement of the scheme which ensures no interface dissipation in case of incompatible time steps but the proposed method is complex to implement and heavy in terms of a computaytional costs. Morevoer it is limited to Newmark integration schemes which have limited quality in case of non linear applications. This paper gives a general framework which allows to couple any one step integration scheme with incompatible time steps. The paper is organised in three parts : the first sections gives the basic theoretiacl framework of the coupling formulation, the second shows its application to some specific integration schemes and the third is devoted to application examples.

\section{Weak formulation of dynamic equilibrium}

Let us now describe the weak formulation of dynamic equilibrium of mechanical systems. We first consider one sub domain, then write the link conditions and extend the formulation to sub domain coupling. 


\subsection{One sub domain}

Let us integrate the virtual power on the time slab $[0, t]$. One gets :

$$
\left\{\begin{array}{r}
W^{*}(t)=\int_{0}^{t}\left(-\int_{\Omega} \operatorname{Tr}\left[\underline{\underline{\sigma}}(P, \tau) \underline{\underline{\epsilon}}\left(\underline{v}^{*}\right)\right] d \Omega\right) \\
+\left(\int_{\Omega} \underline{v}^{* T}\left[\underline{f}^{v o l}(P, \tau)-\rho \underline{\ddot{u}}(P, \tau)\right] d \Omega\right) \\
+\left(\int_{\partial \Omega_{t}} \underline{v}^{* T} \underline{F} d S\right) d \tau=0 .
\end{array}\right\} \forall v^{*}
$$

In the preceding Equation [1] $\Omega$ is the domain, $\underline{v}^{*}$ the virtual velocity field, $\mathrm{P}$ the considered point in the domain, $\tau$ the integration time, $\underline{\underline{\sigma}}$ the stress tensor, $\rho$ the specific mass, $\underline{\ddot{u}}$ the acceleration, $f^{v o l}$ the body forces, and $\underline{\bar{F}}$ the prescribed forces on the boundary. If one now introduces a spatial discretization (e.g. Finite elements) one gets :

$$
W^{*}(t)=\int_{0}^{t} \mathbf{v}^{*}(\tau)^{T}\left[\mathbf{F}^{\mathbf{i n t}}(\tau)+\mathbf{M} \ddot{\mathbf{u}}(\tau)-\mathbf{F}^{\mathbf{e x t}}(\tau)\right] d \tau
$$

In Equation [2] $\mathbf{F}^{\text {int }}$ is the internal force vector, $\mathbf{F}^{\mathbf{e x t}}$ the external loads, $\mathbf{M}$ the mass matrix. One now imposes the kinematic constraints by the means of Lagrange multiplier link forces $\lambda(\tau)$. Equation[2] becomes :

$$
W_{L}^{*}(t)=W^{*}(t)+\int_{0}^{t}(\mathbf{v}(\tau))^{T} \lambda^{*}(\tau) d \tau+\int_{0}^{t}(\lambda(\tau))^{T} \mathbf{v}^{*}(\tau) d \tau
$$

Let us observe that the link forces have a similar role than the external loads and that their dual variable is the velocity field.

Let us now observe the time integration of Equation [3] on a finite time step $\mathrm{h}$ ( $h=$ $\left.t_{n+1}-t_{n}\right)$. Let us denote $\Delta(x)$ the finite increment of a variable $\mathrm{x}$ on time step $\mathrm{h}$. The contribution of time step h into Equation [3] is :

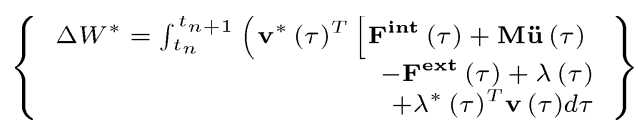

If we now suppose that the time functions $\mathbf{F}^{\mathbf{i n t}}(\tau), \ddot{\mathbf{u}}(\tau), \mathbf{F}^{\operatorname{ext}}(\tau), \mathbf{v}(\tau)$ et $\lambda(\tau)$ are constant in the time step $\mathrm{h}$, the equilibrium check is equivalent to the minimization of the virtual power increment $\Delta W^{*}$. The equilibrium equations are not verified in a strong sens on the whole time interval $h$, but only in a weak sense. Nevertheless this is the basic hypothesis of all usual time integrators (Newmark (Newmark, 1959), HHT (Hilber, 1977), Simo(Simo, 1991), Krenk (Krenk, n.d.) et Verlet (Verlet, 1967) (in its velocity presentation the Verlet's integration is identical to the Explicit Newmark integration scheme)). The link forces $\lambda$ shall be supposed to be constant within the time step $h$. The mean value theorem allows to state that if a function is continuous on the time step $\mathrm{h}$ there exist an (unknown) instant $t_{n+\theta} \in\left[t_{n}, t_{n+1}\right]$ within this time interval for which the dynamic equilibrium equations are exactly verified. The precise definition of this instant depends on the type of integration scheme. Let us now denote $\bar{x}$ the mean value of variable $\mathrm{x}$. One has :

$$
\overline{\Delta W^{*}}=\left|\int_{t_{n}}^{t_{n+1}} \mathbf{v}^{*}(\tau)^{T} d \tau\right|\left[\overline{\mathbf{F}^{\mathbf{i n t}}}+\mathbf{M} \overline{\mathbf{u}}-\overline{\mathbf{F}^{\mathbf{e x t}}}+\mathbf{L} \bar{\lambda}\right]+\left|\int_{t_{n}}^{t_{n+1}} \mathbf{v}(\tau)^{T} d \tau\right|\left(\mathbf{L} \overline{\lambda^{*}}\right)
$$




\subsection{Two sub domains coupling}

Let us couple two sub domains A and B glued by interface $\Gamma$. The link conditions shall impose the same velocities on both sides of the interface. Let us set:

$$
\begin{aligned}
& \mathbf{v}_{\mathbf{A}}^{\Gamma}=\mathbf{L}_{\mathbf{A}} \mathbf{v}_{\mathbf{A}} \\
& \mathbf{v}_{\mathbf{B}}^{\Gamma}=\mathbf{L}_{\mathbf{B}} \mathbf{v}_{\mathbf{B}}
\end{aligned}
$$

The increment of virtual power on time step $\mathrm{h}$ writes :

$$
\left(\overline{\Delta W^{*}}\right)^{t o t}=\left(\overline{\Delta W^{*}}\right)^{A}+\left(\overline{\Delta W^{*}}\right)^{B}+\left(\overline{\Delta W^{*}}\right)_{A B}^{l i n k}
$$

The power variation associated with link forces is :

$$
\left(\overline{\Delta W^{*}}\right)_{A B}^{l i n k}=h\left(\mathbf{L}_{\mathbf{A}} \overline{\mathbf{v}_{\mathbf{A}}}+\mathbf{L}_{\mathbf{B}} \overline{\mathbf{v}_{\mathbf{B}}}\right)^{T} \overline{\lambda_{\mathbf{A B}}^{*}}
$$

Equation [8] implies that if the velocities verify the kinematic constraint the variation of link power is zero and the inverse assertion is also true. One immediately concludes that this formulation ensures that interface energy is strictly zero. The system energy is then exactly equal to the sum of the energies of the parts.

This simple idea is the key of the proposed method.

- remark $1:$ No hypothesis has been done on the time integration scheme in each sub domain : it can be then concluded that the proposed method is very general and does not depend on the time integration scheme provided that it permits to pass from state $\mathrm{n}$ to state $\mathrm{n}+1$ in one step.

- remark 2 : The stability of the system is only governed by the stability of each part because the interface energy is exactly zero.

- remark 3 : No hypothesis has been done on the time variation of the link forces. They are piecewise constant functions on each time step. Their value at time steps $t_{n}$ for instance must be interpolated between the values on time slab $n-1$ and $n$.

- remark 4 : The time weak link conditions is such that the time histories of displacement fields on both sides of the interface may be different but their mean value is the same on each time step. This is due to the following equation :

$$
\int_{t_{n}}^{t_{n+1}}\left(\mathbf{L}_{\mathbf{A}} \mathbf{v}_{\mathbf{A}}(\tau)+\mathbf{L}_{\mathbf{B}} \mathbf{v}_{\mathbf{B}}(\tau)\right) d \tau=0 .
$$

- remark 5 : The extension of the proposed method to any number of sub domains is obvious though rather technical.

\section{Time integration schemes}

Let us now present a unique vision of all usual time integrator (HHT, Newmark, SIMO, Krenk and Verlet). The incremental form shall be presented with the velocity 
variable. The incremental dynamic equilibrium equations at time step $n+1$ (including link forces) write :

$$
\begin{aligned}
& \left(\frac{1}{h \gamma} \mathbf{M}+h \frac{\beta}{\gamma}(1+\alpha) \mathbf{K}\right) \Delta \mathbf{v}+\mathbf{L}^{T} \bar{\lambda}=\eta \mathbf{f}_{n+1}+\mu \mathbf{f}_{n} \\
& -(\eta+\mu) \mathbf{K} \mathbf{u}_{n}-h(1+\alpha) \mathbf{K} \mathbf{v}_{n} \\
& +(\eta-\mu)\left(\left(\frac{1}{\gamma}-1\right) \mathbf{M}+(1+\alpha)\left(\beta-\frac{\gamma}{2}\right) h^{2} \mathbf{K}\right) \mathbf{a}_{n}
\end{aligned}
$$

$$
\mathbf{L} \Delta \mathbf{v}=\mathbf{0}
$$

5 parameters which allow to define any scheme in the same format are defined in Table 1:

\begin{tabular}{|c|c|c|c|c|c|}
\hline integrator type & HHT & Newmark & SIMO & Verlet & Krenk \\
\hline$\alpha$ & $\alpha$ & 0. & 0. & 0. & 0. \\
\hline$\beta$ & $\beta$ & $\beta$ & $\frac{1}{4}$ & 0. & $\frac{1}{4}$ \\
\hline$\gamma$ & $\gamma$ & $\gamma$ & $\frac{1}{2}$ & $\frac{1}{2}$ & $\frac{1}{2}$ \\
\hline$\eta$ & $1+\alpha$ & 1 & 1 & 1 & 1 \\
\hline$\mu$ & $-\alpha$ & 0 & 1 & 0 & 1 \\
\hline
\end{tabular}

Table 1 - Parameter values for any time integration scheme

The equivalent stiffness $\mathbf{K}_{*}$ writes :

$$
\mathbf{K}_{*}=\mathbf{M} \frac{1}{\gamma h}+(1+\alpha) \frac{\beta}{\gamma} h \mathbf{K}
$$

The equations system [22] becomes :

$$
\left[\begin{array}{cc}
\mathbb{K}_{*} & \mathbb{L}^{T} \\
\mathbb{L} & \mathbf{0}
\end{array}\right]\left[\begin{array}{c}
\Delta \mathbb{U} \\
\bar{\lambda}
\end{array}\right]=\left[\begin{array}{c}
\mathbb{F} \\
\mathbf{0}
\end{array}\right]-\left[\begin{array}{cc}
\mathbb{N} & \mathbf{0} \\
\mathbf{0} & \mathbf{0}
\end{array}\right]\left[\begin{array}{c}
\mathbb{U}_{n} \\
\mathbf{0}
\end{array}\right]
$$

where

$$
\begin{aligned}
& \mathbb{K}_{*}=\left[\begin{array}{ccc}
\mathbf{K}_{*} & \mathbf{0} & \mathbf{0} \\
-\frac{\beta h}{\gamma} \mathbf{I} & \mathbf{I} & \mathbf{0} \\
-\frac{1}{\gamma h} \mathbf{I} & \mathbf{0} & \mathbf{I}
\end{array}\right], \mathbb{L}^{T}=\left[\begin{array}{c}
\mathbf{L}^{T} \\
\mathbf{0} \\
\mathbf{0}
\end{array}\right] \\
& \Delta \mathbb{U}=\left[\begin{array}{c}
\Delta \mathbf{v} \\
\Delta \mathbf{u} \\
\Delta \mathbf{a}
\end{array}\right], \bar{\lambda}=\left[\begin{array}{l}
\bar{\lambda} \\
\mathbf{0} \\
\mathbf{0}
\end{array}\right]
\end{aligned}
$$


and

$$
\begin{aligned}
& \mathbb{F}=\left[\begin{array}{c}
\eta \mathbf{f}_{n+1}+\mu \mathbf{f}_{\mathbf{n}} \\
\mathbf{0} \\
\mathbf{0}
\end{array}\right], \mathbb{U}_{n}=\left[\begin{array}{c}
\mathbf{v}_{n} \\
\mathbf{u}_{n} \\
\mathbf{a}_{n}
\end{array}\right] \\
& \mathbb{N}=\left[\begin{array}{ccc}
-(1+\alpha) h \mathbf{K} & -(\eta+\mu) \mathbf{K} & (\eta-\mu)\left[\left(\frac{1}{\gamma}-1\right) \mathbf{M}+(1+\alpha)\left(\beta-\frac{\gamma}{2}\right) h^{2} \mathbf{K}\right] \\
h \mathbf{I} & 0 & \left(\frac{\gamma}{2}-\beta\right) h^{2} \mathbf{I} \\
\mathbf{0} & 0 & \frac{1}{\gamma} \mathbf{I}
\end{array}\right]
\end{aligned}
$$

The displacement increment is the sum of a free part (computed without link force) and of a link displacement due to the constraints (links) applications :

$$
\Delta \mathbb{U}=\Delta \mathbb{U}_{\text {free }}+\Delta \mathbb{U}_{\text {link }}
$$

One uses recurrence formulas to step from state $n$ to state $n+1$. These formulas except for Krenk write :

$$
\begin{aligned}
& \mathbf{u}_{n+1}=\mathbf{u}_{n}+h \mathbf{v}_{n}+\left(\frac{1}{2}-\beta\right) h^{2} \mathbf{a}_{n}+\beta h^{2} \mathbf{a}_{n+1} \\
& \mathbf{v}_{n+1}=\mathbf{v}_{n}+(1-\gamma) h \mathbf{a}_{n}+\gamma h \mathbf{a}_{n+1}
\end{aligned}
$$

where $\gamma$ and $\beta$ are the usual Newmark scheme parameters. For Krenk's scheme these recurrence equations are :

$$
\begin{aligned}
& \mathbf{u}_{n+1}=\mathbf{u}_{n}+h \mathbf{v}_{n}+\frac{h}{2} \Delta \mathbf{v} \\
& \mathbf{v}_{n+1}=\mathbf{v}_{n}+\Delta \mathbf{v}
\end{aligned}
$$

The stability conditions of HHT scheme is a known function of $\alpha, \beta$ and $\gamma$ values ((Geradin, 1997)). No stability demonstration is available for non linear applications. Simo and Verlet have the same stability properties as Newmark for linear applications but these first two schemes are also proven to be stable for non linear applications. Krenk has the same stability properties as Simo scheme.

\section{Sub-domain coupling}

Link forces have to be added on the common boundaries to "glue" the subdomains. They are described on Figure(1). Each sub-domain (or code) k uses its own integration 


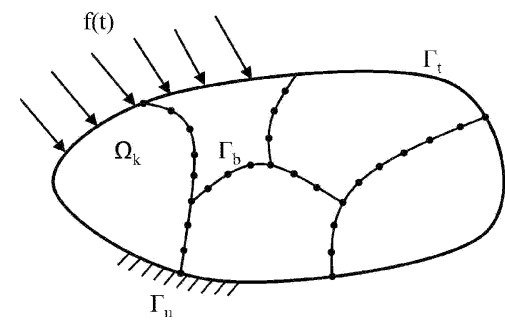

(a)

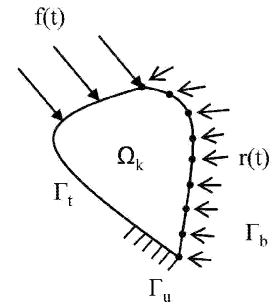

(b)

Figure 1 - Sub-domain gluing

scheme k. The link forces are Lagrange multipliers. The equilibrium equations for sub domain $\mathrm{k}$ are given by :

$$
\left\{\begin{array}{r}
\left(\frac{1}{h \gamma_{k}} \mathbf{M}_{k}+h \frac{\beta_{k}}{\gamma_{k}} \mathbf{K}_{k}\right) \Delta \mathbf{v}^{k}+\mathbf{L}_{k}^{T} \bar{\lambda}=\eta_{k} \mathbf{f}_{n+1}^{k}+\mu_{k} \mathbf{f}_{n}^{k} \\
-\left(\eta_{k}+\mu_{k}\right) \mathbf{K}_{k} \mathbf{u}_{n}^{k}-h \mathbf{K}_{k} \mathbf{v}_{n}^{k} \\
+\left(\left(\eta_{k}-\mu_{k}\right)\left(\frac{1}{\gamma_{k}}-1\right) \mathbf{M}_{k}-\left(\frac{\gamma_{k}}{2}-\beta_{k}\right) h^{2} \mathbf{K}_{k}\right) \mathbf{a}_{n}^{k}
\end{array}\right\}
$$

Let us now study the coupling between sub domains A and B having the same time step h. In a second step one shall present the case for which the time steps are different.

\subsection{Same time step}

The time steps are the same but the time steps are different. One has then to solve :

$$
\left\{\begin{array}{r}
\left(\frac{1}{h \gamma_{A}} \mathbf{M}_{A}+h \frac{\beta_{A}}{\gamma_{A}}\left(1+\alpha_{A}\right) \mathbf{K}_{A}\right) \Delta \mathbf{v}^{A}+\mathbf{L}_{A}^{T} \bar{\lambda}=\eta_{A} \mathbf{f}_{n+1}^{A}+\mu_{A} \mathbf{f}_{n}^{A} \\
-\left(\eta_{A}+\mu_{A}\right) \mathbf{K}_{A} \mathbf{u}_{n}^{A}-h\left(1+\alpha_{A}\right) \mathbf{K}_{A} \mathbf{v}_{n}^{A} \\
+\left(\eta_{A}-\mu_{A}\right)\left[\left(\frac{1}{\gamma_{A}}-1\right) \mathbf{M}_{A}-\left(1+\alpha_{A}\right)\left(\frac{\gamma_{A}}{2}-\beta_{A}\right) h^{2} \mathbf{K}_{A}\right] \mathbf{a}_{n}^{A} \\
\left(\frac{1}{h \gamma_{B}} \mathbf{M}_{B}+h \frac{\beta_{B}}{\gamma_{B}}\left(1+\alpha_{B}\right) \mathbf{K}_{B}\right) \Delta \mathbf{v}^{B}+\mathbf{L}_{B}^{T} \bar{\lambda}=\eta_{B} \mathbf{f}_{n+1}^{B}+\mu_{B} \mathbf{f}_{n}^{B} \\
-\left(\eta_{B}+\mu_{B}\right) \mathbf{K}_{B} \mathbf{u}_{n}^{B}-h\left(1+\alpha_{B}\right) \mathbf{K}_{B} \mathbf{v}_{n}^{B} \\
+\left(\eta_{B}-\mu_{B}\right)\left[\left(\frac{1}{\gamma_{B}}-1\right) \mathbf{M}_{B}-\left(1+\alpha_{B}\right)\left(\frac{\gamma_{B}}{2}-\beta_{B}\right) h^{2} \mathbf{K}_{B}\right] \mathbf{a}_{n}^{B} \\
L_{A} \Delta \mathbf{v}^{A}+L_{B} \Delta \mathbf{v}^{B}=0
\end{array}\right\}
$$


The matrix expression is then :

$$
\left[\begin{array}{ccc}
\mathbb{K}_{A}^{*} & 0 & \mathbb{L}^{T} \\
0 & \mathbb{K}_{B}^{*} & \mathbb{L}_{B}^{A} \\
\mathbb{L}_{A} & \mathbb{L}_{B} & 0
\end{array}\right]\left[\begin{array}{c}
\Delta_{\mathbb{U}}^{A} \\
\Delta_{\bar{U}}^{B} \\
\bar{\lambda}
\end{array}\right]=\left[\begin{array}{c}
\mathbb{F}^{A} \\
\mathbb{F}^{B} \\
\mathbf{0}
\end{array}\right]-\left[\begin{array}{ccc}
\mathbb{N}_{A} & \mathbf{0} & \mathbf{0} \\
\mathbf{0} & \mathbb{N}_{B} & \mathbf{0} \\
\mathbb{L}_{A} & \mathbb{L}_{B} & \mathbf{0}
\end{array}\right]\left[\begin{array}{c}
\mathbb{U}_{n}^{A} \\
\mathbb{U}_{n}^{B} \\
\mathbf{0}
\end{array}\right]
$$

The same method as for a single sub domain can be applied. One solves the "free" equilibrium for each sub domain : one hence gets the "free" kinematic quantities. One then compute the Lagrange multiplier which enforce the kinematic continuity. One then computes in each sub domain the link kinematic quantities which are added to the "free" ones to get the problem solution. Steps 1 and 3 are native parallel.

\subsection{Different time steps}

One shall suppose that time step of sub domain A is an integer multiple of the one of sub domain B :

$$
h_{A}=m h_{B}
$$

Let us consider $\mathrm{j}$ one of the time steps of sub domain $\mathrm{B}$ defined by $(j \in[1, m-1])$. Let us set :

$$
\overline{\Delta \mathbf{x}}=\left(\mathbf{x}_{j+1}-\mathbf{x}_{j}\right), \overline{\overline{\mathbf{x}}}=\int_{j h_{B}}^{(j+1) h_{B}} x(\tau) d \tau
$$

Let us now suppose that the Lagrange multiplier (link force) $\overline{\bar{\lambda}}_{B}^{j}$ is constant for all j sub steps $h_{B}$. Its value is denoted à $\bar{\lambda}_{B}$ and is constant during time step $h_{A}$. This leads to :

$$
\Delta W_{A B}^{\text {interface }}=\left(\left(\Delta \mathbf{v}^{A}\right)^{T} \mathbf{L}_{A}^{T}+\sum_{j=1}^{m}\left(\Delta \mathbf{v}_{j}^{B}\right)^{T} \mathbf{L}_{B}^{T}\right) \bar{\lambda}
$$

The kinematic constraints on the velocities write :

$$
\mathbf{L}_{A} \Delta \mathbf{v}^{A}+\mathbf{L}_{B} \Delta \mathbf{v}^{B}=0
$$

which also writes :

$$
\mathbf{L}_{A} \Delta \mathbf{v}^{A}+\sum_{j=1}^{m} L_{B} \Delta \mathbf{v}_{j}^{B}=0
$$

This leads to a zero interface work. For each macro time step the equilibrium equation is :

$$
\mathbb{K}_{A}^{*} \Delta \mathbb{U}^{A}+\mathbb{L}_{A}^{T} \bar{\lambda}+\mathbb{N}_{A} \mathbb{U}^{A}=\mathbb{F}_{A}
$$


For each micro(small) time step $\mathrm{j}$ the equilibrium equation is :

$$
\mathbb{K}_{B}^{*} \Delta\left(\mathbb{U}_{j}^{B}\right)+\mathbb{L}_{B}^{T} \bar{\lambda}+\mathbb{N}_{B} \mathbb{U}_{j}^{B}=\mathbb{F}_{B}
$$

The generalized unknown vector $\left(\Delta \mathbb{U}_{j}^{B}\right.$ and $\left.\mathbb{U}_{j}^{B}\right)$ for each small time step on sub domain $B$ are simply the summation of preceding (j-1) sub step vectors :

$$
\Delta \mathbb{U}_{j}^{B}=\sum_{i=1}^{j-1} \Delta \mathbb{U}_{i}^{B} \text { and } \mathbb{U}_{j}^{B}=\mathbb{U}_{0}^{B}+\sum_{i=1}^{j-2} \Delta \mathbb{U}_{i}^{B}
$$

After some lengthy calculation one get the following matrix system to solve :

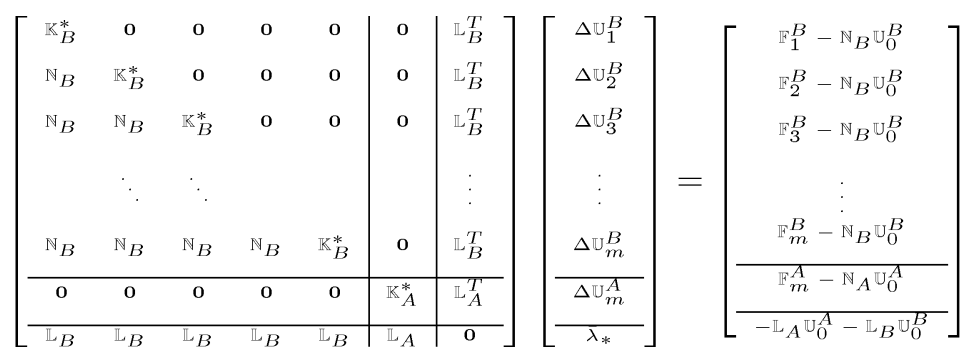

This manner to express the problem shows that the implementation is not very difficult. One observes that to get the free kinematic quantities one performs one step in sub domain $\mathrm{A}$ and in the meantime one performs $\mathrm{m}$ successive resolutions in sub domain B. The free solution increment on sub domain B is simply the sum of the $m$ sub increments. One then solves the interface problem to get the link forces between the sub domains. One finally computes the so called link kinematic variables in the same manner as for the the free quantities. This algorithm is naturally parallel and no communication between A and B is necessary during the sub steps. This method does no generate any interface energy during the large time step even in case of incompatible time steps.It is simpler than the Prakash method. It is also more general because it applies for any one step time integrator.

\section{Application examples}

\subsection{The plate case}

One considers the wave propagation in a square plate of side $L=1$. The Young is $E=1$, the specific mass is $\rho=1$. The Poisson's ratio is $\mu=0.3$. The loading is a step load applied to the top right corner of the plate. The plate is fixed at its right bottom corner. 


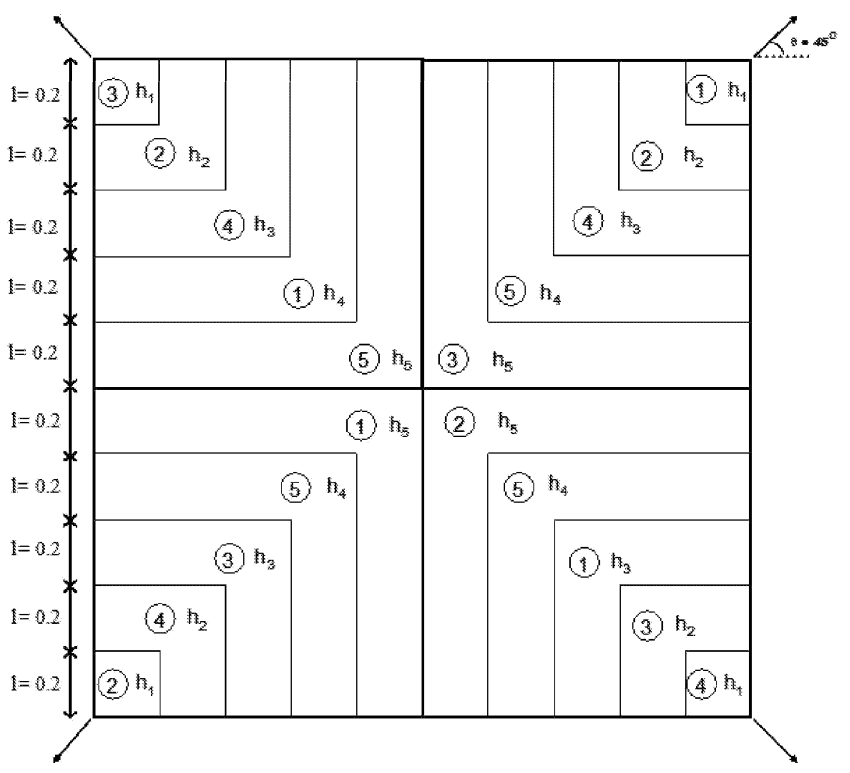

Figure 2 - 20 sub domains partition with 5 different integration schemes ( the type of integrator is displayed in each circle)

The mesh used 4 node finite elements in plane strain. Figure (2) shows the sub domain decomposition each of the having its own time step. The scheme parameters are given in Table 2. 5 time integrators with 5 different time steps are combined : $h_{i}=m_{i} h_{1} \forall i \in\{1 . .5\}$ the smallest one being $h_{1}=0.016$. Let is also observe that spatial meshes are incompatible at the interfaces : a kinematic interface continuity constraint is chosen here. The computation uses 100 small time steps. The interface work value (Figure 3 ) is about $10^{-15}$. The total strain energy being close to 1 one observes a zero interface energy.

\begin{tabular}{|c|c|c|c|}
\hline Scheme $^{\circ}$ & $\alpha$ & $\gamma$ & $\beta$ \\
\hline 1 & 0.0 & 0.5 & 0.25 \\
\hline 2 & 0.0 & 0.5 & 0.66 \\
\hline 3 & 0.0 & 0.6 & 0.3025 \\
\hline 4 & -0.2 & 0.7 & 0.36 \\
\hline 5 & 0.0 & 0.55 & 0.27565 \\
\hline
\end{tabular}

Table 2 - Parameters used in the simulation, $\alpha, \gamma$ and $\beta$ 


\begin{tabular}{|c|c|c|c|c|}
\hline$m_{1}$ & $m_{2}$ & $m_{3}$ & $m_{4}$ & $m_{5}$ \\
\hline 1 & 2 & 4 & 8 & 16 \\
\hline
\end{tabular}

Table 3 - Time step ratios used

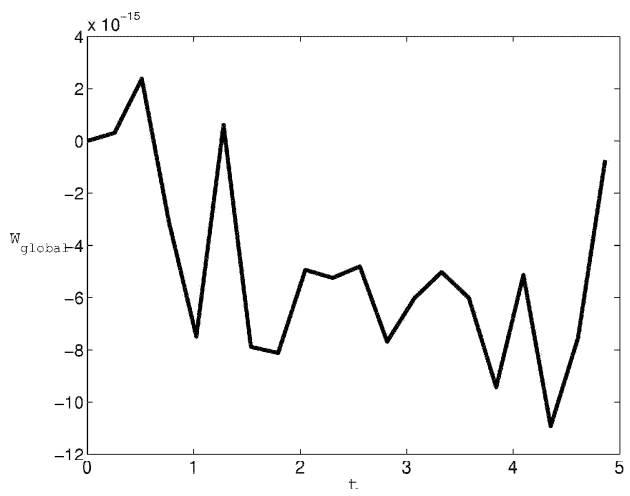

Figure 3 - Interface Work $W_{\text {global }}$

\subsection{A Pipe in elasto plasticity}

A pipe meshed in shell elements is the subjected to a step bending load of $210^{5} \mathrm{~N}$ at one extremity and clamped at the other one. The pipe has a radius of $1 \mathrm{~m}$ and a length of $8 \mathrm{~m}$ it is $0.5 \mathrm{~cm}$ thick. It is stiffened by 4 equally spaced axial and 4 radial stiffeners placed every $2 \mathrm{~m}$ the first and last one being at $1 \mathrm{~m}$ from the extremities. All stiffeners are $0.1 \mathrm{~m}$ high and $0.5 \mathrm{~cm}$ thick. Figure[4) displays the pipe. It is made of steel (Young's modulus 200000MPa, Poisson's ratio 0.3, Yield stress 200MPa, density 8). It is meshed with DKT shell element and has about 8700 nodes. It is decomposed in six subdomains.

The black and white subdomains (close to the clamped part) are computed using the average acceleration Newmark scheme. The two grey subdomains (in the middle of 


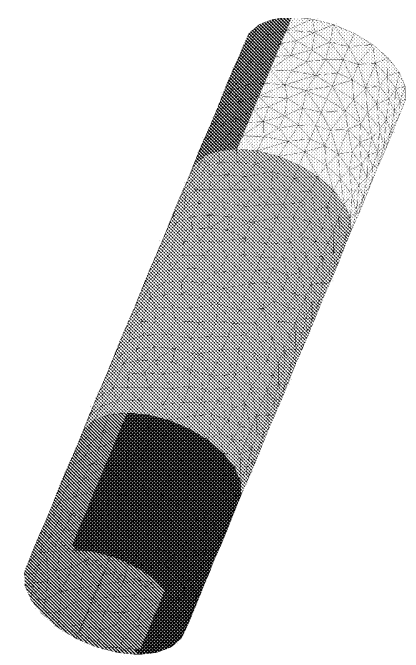

Figure 4 - Mesh of the pipe and its sub domains

the pipe) use on the one hand the mid point integration rule and on the other hand the Krenk's integration scheme. The material is supposed to be linear elastic in these 4 sub domains. The two other subdomains (close to the loaded part) are computed using the dynamic explicit Newmark time integrator and are non linear. The 4 implicit sub domains use a time step which is 100 times higher than the two explicit sub domains. The results are displayed in Figure(5) for $m=100$. The response is rather good and the interface energy has been computed to be less than $10^{-10}$ of the maximum strain energy.

\section{Conclusion}

This papers gives a simple method to construct assemblies of time integrators which do not dissipate at the interface. It relies on a simple basic weak vision of time integration of equilibrium equations. The schemes allow to have some freedom to the 

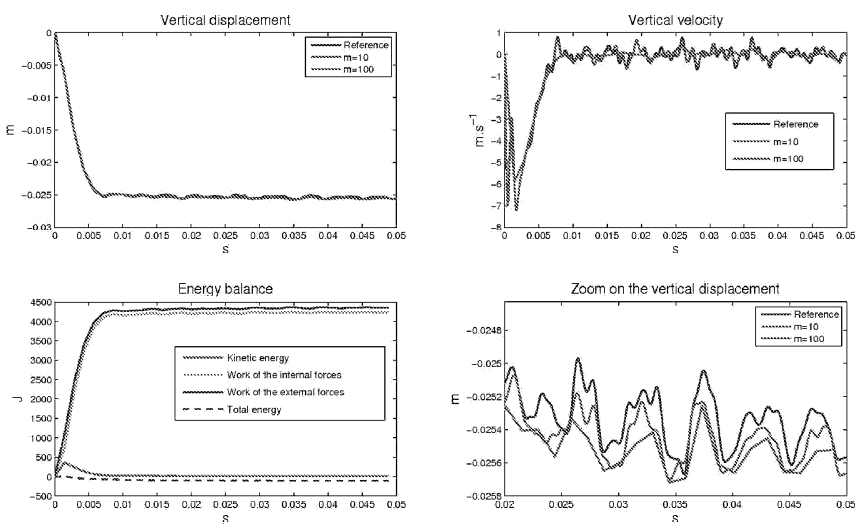

Figure 5 - Results of sub domain pipe computation with $m=100$

kinematic interface variables which are not identical during the whole time step. These schemes can be extended to non linear dynamic computations. One should study with more care how does this type of interface treatment behaves in the frequency domain. For instance which frequency distortions this type of interface treatment induces.

\section{Bibliography}

Champaney Cognard J-Y D. D. L. P., « Large scale applications on parallel computers of a mixed domain decomposition method », Computer methods in applied mechanics and engineering, vol. 19, p. 253-263, 1997.

Combescure Gravouil A., « A numerical scheme to couple subdomains with different time-steps for predominantly linear transient analysis », Computer methods in applied mechanics and engineering, vol. 191, p. 1129-1157, 2002.

Dhia B., « Multiscale mechanical problems : the Arlequin method», Comptes Rendus de l'Académie des Sciences - Series IIB - Mechanics-Physics-Astronomy, vol. 12, p. 899-904, 1998.

Farhat Crivelli L G. M., « On the spectral stability of time integration algorithms for a class of constrained dynamics problems. », Collection of Technical PapersÂAIAA/ASME Structures, Structural Dynamics and Materials Conferencep. 80-97, 1993.

Farhat Crivelli L R. F.-X., « Transient FETI methodology for large-scale parallel implicit computations in structural mechanics. », International Journal for Numerical Methods in Engineering, vol. 37, p. 1945-1975, 1994.

Geradin R. D., Mechanical Vibrations, Theory and Applications to Structural Dynamics, Wiley, Chichester, 1997.

Gravouil C. A., « A multi-time-step explicit-implicit method for non-linear structural dynamics », International Journal for Numerical Methods in Engineering, vol. 50, p. 199-225, 2()() 1 . 
Hilber Hughes TJR T. R., « Improved numerical dissipation for time integration algorithms in structural dynamics », Earthquake Engineering and Structural Dynamics, vol. 5, p. 283292, 1977.

Krenk, « Energy conservation and high-frequency damping in numerical time integration », COMPDYN 2007 Conference procedings, n.d.

Ladeveze N. A., « On a multiscale computational strategy with time and space homogenization for structural mechanics », Computer methods in applied mechanics and engineering, vol. 192, p. 3061-3087, 2003.

Newmark, « A method of computation for structural dynamics », Journal of the Engineering Mechanics Division, ASCEp. 67-94, 1959.

Prakash H. K., « A FETI-based multi-time-step coupling method for Newmark schemes in structural dynamics », International Journal for Numerical Methods in Engineering, vol. 61, p. 2183-2204, 2004.

Simo W. K., « Unconditionally stable algorithms for rigid body dynamics that exactly preserve energy and momentum. », International Journal for Numerical Methods in Engineering, vol. 31, p. 19-52, 1991.

Verlet, « A time integration algorithm for dynamics », Phys. Rev, vol. 165, n 201, p. 67-94, 1967. 